\title{
Adaptive error estimation of the Trefftz method for solving the Cauchy problem
}

\author{
C.-T. Chen ${ }^{1}$, K.-H. Chen ${ }^{2}$, J.-F. Lee ${ }^{1} \&$ J.-T. Chen ${ }^{3}$ \\ ${ }^{1}$ Department of Hydraulic and Ocean Engineering, \\ National Cheng Kung University, Tainan, Taiwan \\ ${ }^{2}$ Department of Information Management, Toko University, \\ Chia-yi, Taiwan \\ ${ }^{3}$ Department of Harbor and River Engineering, \\ National Taiwan Ocean University, Keelung, Taiwan
}

\begin{abstract}
In this paper, the Laplace problem with overspecified boundary conditions is investigated by using the Trefftz method. The main difficulty will appear an obvious divergent result when the boundary condition on an overspecified boundary contaminates artificial errors. The occurring mechanism of the unreasonable result originates from an ill-posed influence matrix. The accompanied ill-posed problem is remedied by using the Tikhonov regularization technique and the linear regularization method respectively, to reconstruct the influence matrix. The optimal parameters of the Tikhonov technique and linear regularization method are determined by adopting the adaptive error estimation technique. The numerical evidence of the Trefftz method is given to verify the accuracy of the solutions after comparison with the results of analytical solution and to demonstrate the validity and instructions of the proposed adaptive error estimation technique. The comparison of the Tikhonov regularization technique and the linear regularization method was also discussed in the example.

Keywords: Trefftz method, adaptive error estimation, Cauchy problem, ill-posed problem, Tikhonov technique, linear regularization method, L-curve concept.
\end{abstract}

\section{Introduction}

In 1926, Trefftz [10] presented the Trefftz method for solving boundary value problems by superimposing the basis functions satisfying the governing 
equation, although various versions of the Trefftz method, e.g. direct formulations [8] and indirect formulations [7], have been developed. The unknown coefficients are determined by satisfying the boundary condition with the approximate solution. Many applications to the Helmholtz equation [4], the Navier equation $[15,17]$ and the biharmonic equation [6] had been done. Until recent years, the ill-posed nature in the method was noticed $[8,19]$ increasingly.

The ill-posed nature (behavior) may be one of the following inverse problems or their combinations: (1) lack the determination of the domain, its boundary, or an unknown inner boundary; (2) lack inference of the governing equation; (3) lack identification of boundary conditions and/or initial conditions (the Cauchy problem); (4) lack determination of the material properties involved; (5) lack determination of the forces or inputs acting in the domain [12]. The Cauchy problem is surveyed in this paper.

Sometimes, unreasonable results occur in the Cauchy problem subjected to the measured and contaminated errors on the overspecified boundary condition because of the ill-posed behavior in linear algebraic system $[13,16]$. Mathematically speaking, the Cauchy problem is ill-posed since the solution is very sensitive to the given data. Such a divergent problem could be avoided by using regularization methods $[1,5,14,18]$, e.g., the Tikhonov regularization technique [1] and the linear regularization method [5]. The Tikhonov regularization technique and the linear regularization method had been successfully applied to overcome the ill-posed problem of the Laplace equation $[3,9]$ and to treat with the divergent problems, since the two methods can obtain the convergent solution more stably and reasonably. In this paper, the comparison of two regularization techniques is made to obtain a better method.

For the Cauchy problem, the influence matrix is often ill-posed such that the regularization technique which regularizes the influence matrix is necessary. Both the Tikhonov technique and the linear regularization method transform into well-posed ones by choosing appropriate parameters for $\lambda$ and $\lambda^{*}$, respectively [2]. The optimal parameter can be determined according to a local minimal point at error curve (similarly with L-curve shape) by implementing the adaptive error estimation technique. The corner (local minimal point) of the Lcurve determines the optimal value of $\lambda$ which provides the least relative error. The proposed error estimation technique belongs to an adaptive technique and does not need to compare the results with analytical solution. It will be elaborated latterly.

The purpose of this paper is to deal with the Cauchy problem with ill-posed nature of numerical instability by implementing the Trefftz Method in conjunction with the Tikhonov technique, linear regularization method, and adaptive error estimation technique. The technique is employed to obtain the optimal parameter to remedy the ill-posed behavior. Finally, the results of the example contaminated with artificial noise on the overspecified boundary condition are given to illustrate the validity of the proposed technique. Good agreements are observed as comparing analytical solutions. Under no analytical solutions, the numerical examples also demonstrate the validity and instructions of the proposed adaptive error estimation technique. 


\section{Formulation}

\subsection{Governing equation and over-specified boundary condition}

We consider the inverse problem for Laplace equation with overspecified boundary condition as shown in Fig. 1, which satisfies:

$$
\nabla^{2} u(\underset{\sim}{x})=0, \underset{\sim}{x} \in D
$$

subjected to the boundary condition on $B_{1}$ as

$$
u_{1}(\underset{\sim}{x})=\bar{u}_{1}(\underset{\sim}{x}), t_{1}(\underset{\sim}{x})=\bar{t}_{1}(\underset{\sim}{x}), \underset{\sim}{x} \in B_{1}
$$

where $\nabla^{2}$ is the Laplacian operator, $D$ is the domain of interesting, $t(\underset{\sim}{x})=\frac{\partial u(\underset{\sim}{x})}{\partial n_{x}}$ in which $n_{x}$ is the normal vector at $\underset{\sim}{x}, B$ is the whole boundary which consists of the known boundary $\left(B_{1}\right)$ and the unknown boundary $\left(B_{2}\right)$.

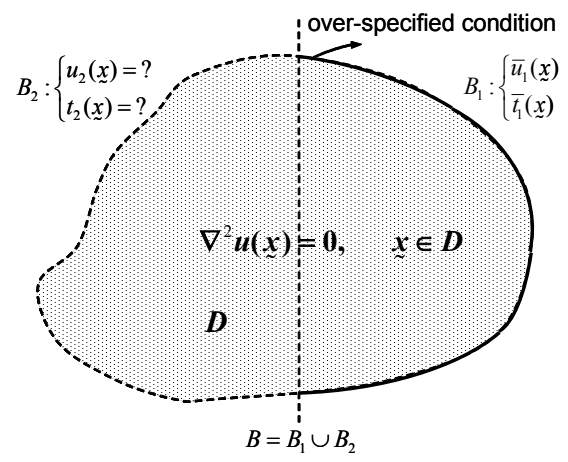

Figure 1: Sketch diagram of inverse problem with over-specified condition.

\subsection{Methodology}

\subsubsection{The Trefftz method}

In the Trefftz method, the field solution $u(\underset{\sim}{x})$ is superimposed by the T-complete functions, $A_{j}(\underset{\sim}{x})$, as follows:

$$
u(\underset{\sim}{x})=\sum_{j=1}^{2 N} w_{j} A_{j}(\underset{\sim}{x})
$$

where $2 \mathrm{~N}+1$ is the number of T-complete functions, $w_{j}$ is the unknown coefficient, $A_{j}(\underset{\sim}{x})$ is the T-complementary set which satisfies the Laplace equation. For the interior problem, we choose $1, \rho^{n} \sin (n \theta)$ and $\rho^{n} \cos (n \theta)$ $(n \in N)$, to be the bases of the complementary set in two-dimensional problem. Therefore, the eqn (3) can be expressed by 


$$
u(\rho, \theta)=a_{0}+\sum_{n=1}^{N-1} a_{n} F_{n}(\rho, \theta)+\sum_{n=1}^{N} b_{n} G_{n}(\rho, \theta)
$$

where $F_{n}(\rho, \theta)=\rho^{n} \cos (n \theta)$ and $G_{n}(\rho, \theta)=\rho^{n} \sin (n \theta)$. And the normal differential of the field solution $u(x)=u(\rho, \theta)$, is written as below:

$$
\begin{array}{r}
t(\underset{\sim}{x})=\frac{\partial u(\underset{\sim}{x})}{\partial n_{x}}=\sum_{j=1}^{2 N} w_{j} \frac{\partial A_{j}(\underset{\sim}{)})}{\partial n_{x}}=\sum_{j=1}^{2 N} w_{j} B_{j}(\underset{\sim}{x}) \\
t(\rho, \theta)=\frac{\partial u(\rho, \theta)}{\partial n_{\underline{x}}}=\sum_{n=1}^{N-1} a_{n} F_{n}^{*}(\rho, \theta)+\sum_{n=1}^{N} b_{n} G_{n}^{*}(\rho, \theta)
\end{array}
$$

where

$$
\begin{gathered}
F_{n}^{*}(\rho, \theta)=\left[\sum_{n=1}^{N-1} n \rho^{n-1} \cos (n \theta) \cdot \cos \theta+\sum_{n=1}^{N-1} n \rho^{n} \sin (n \theta) \cdot \frac{\sin \theta}{r}\right] \cdot \vec{n}_{x}, \\
+\left[\sum_{n=1}^{N-1} n \rho^{n-1} \cos (n \theta) \cdot \sin \theta-\sum_{n=1}^{N-1} n \rho^{n} \sin (n \theta) \cdot \frac{\cos \theta}{r}\right] \cdot \vec{n}_{y} \\
G_{n}^{*}(\rho, \theta)=\left[\sum_{n=1}^{N} n \rho^{n-1} \sin (n \theta) \cdot \cos \theta-\sum_{n=1}^{N} n \rho^{n} \cos (n \theta) \cdot \frac{\sin \theta}{r}\right] \cdot \vec{n}_{x} \\
+\left[\sum_{n=1}^{N} n \rho^{n-1} \sin (n \theta) \cdot \sin \theta+\sum_{n=1}^{N} n \rho^{n} \cos (n \theta) \cdot \frac{\cos \theta}{r}\right] \cdot \vec{n}_{y} .
\end{gathered}
$$

In order to obtain the unknown coefficients $w_{j}=a_{0}, a_{n}$ and $b_{n}, \mathrm{~N}$ boundary points on the over-specified boundary $\left(\left(\rho_{1}, \theta_{1}\right),\left(\rho_{2}, \theta_{2}\right), \cdots,\left(\rho_{N}, \theta_{N}\right)\right) \in B_{1}$ are collocated. Eqn (4) and (6) match the boundary condition on the boundary points to obtain the following linear algebraic system

$$
[A]\{x\}=\{b\} \Leftrightarrow\left\{\begin{array}{l}
\left\{\bar{u}_{1}\right\}_{N \times 1} \\
\left\{\bar{t}_{1}\right\}_{N \times 1}
\end{array}\right\}=\left[\begin{array}{l}
{\left[A_{1}\right]_{N \times 2 N}} \\
{\left[B_{1}\right]_{N \times 2 N}}
\end{array}\right]\{w\}_{2 N \times 1},
$$

where

$$
\left\{\bar{u}_{1}\right\}_{N \times 1}=\left\{\begin{array}{c}
\bar{u}_{1} \\
\bar{u}_{2} \\
\vdots \\
\bar{u}_{N}
\end{array}\right\},\left\{\bar{t}_{1}\right\}_{N \times 1}=\left\{\begin{array}{c}
\bar{t}_{1} \\
\overline{t_{2}} \\
\vdots \\
\bar{t}_{N}
\end{array}\right\}
$$




$$
\begin{aligned}
& {\left[A_{1}\right]_{N \times 2 N}=} {\left[\begin{array}{cccccccc}
A_{1,1} & A_{1,2} & \cdots & A_{1, N+1} & A_{1, N+2} & \cdots & A_{1,2 N} \\
\vdots & \vdots & \vdots & \ddots & \vdots & \ddots & \vdots \\
A_{N, 1} & A_{N+1,2} & \cdots & A_{N, N+1} & A_{N, N+2} & \cdots & A_{N, 2 N}
\end{array}\right] } \\
& {\left[B_{1}\right]_{N \times 2 N}=\left[\begin{array}{ccccccc}
0 & B_{1,2} & \cdots & B_{1, N+1} & B_{1, N+2} & \cdots & B_{1,2 N} \\
\vdots & \vdots & \vdots & \ddots & \vdots & \ddots & \vdots \\
0 & B_{N, 2} & \cdots & B_{N, N+1} & B_{N, N+2} & \cdots & B_{N, 2 N}
\end{array}\right] } \\
&\{w\}_{2 N \times 1}=\left\{\begin{array}{l}
a_{0} \\
a_{1} \\
\vdots \\
a_{N-1} \\
b_{1} \\
b_{2} \\
\vdots \\
b_{N}
\end{array}\right\}
\end{aligned}
$$

so that the coefficients $w_{j}=a_{0}, a_{n}$ and $b_{n}$ of numerical solutions can be determined by using linear algebraic system solver.

\subsubsection{Regularization techniques for the Cauchy problem}

2.2.2.1 Tikhonov technique Tikhonov [1] proposed a method to transform this ill-posed problem into a well-posed one. Instead of solving $[A]\{x\}=\{b\}$ directly, the procedures of the Tikhonov technique are written as follows:

$$
\text { (I). Minimize }\|x\|^{2} \text {, subject to }\|A x-b\|^{2} \leq \varepsilon
$$

where $\varepsilon$ is the prescribed error tolerance.

(II). The proposed problem in eqn (12) is equivalent[9] described as below:

$$
\text { minimize }\|x\|^{2} \text {, subject to }\|A x-b\|^{2} \leq \varepsilon^{*},
$$

and the Euler-Lagrange equation obtained from reference [9] can be written as

$$
\left(A^{T} A+\lambda^{(\mathrm{T})} I\right) x=A^{T} b
$$

where $T$ denotes matrix transposition, $\lambda^{(\mathrm{T})}$ is the regularization parameter (Lagrange parameter), in which the subscript (T) denotes the Tikhonov technique. 
2.2.2.2 Linear regularization method The single central idea in the inverse theory is the prescription [5],

$$
\text { minimize: } P[x]+\lambda^{(\mathrm{L})} Q[x]
$$

where $\lambda^{(L)}$ is the regularization parameter, in which the subscript $(\mathrm{L})$ denotes the Linear regularization method, $P[x]=|A \cdot x-b|^{2}>0 \quad$ and $Q[x]=x \cdot H \cdot x>0$ are two positive functions of $x$,

$$
\begin{aligned}
{[\mathrm{H}]_{\mathrm{M} \times \mathrm{M}} } & =[\mathrm{B}]_{\mathrm{M} \times \mathrm{M}-1)}^{T} \cdot[\mathrm{B}]_{(\mathrm{M}-1) \times \mathrm{M}} \\
& =\left(\begin{array}{cccccccccc}
1 & -1 & 0 & 0 & \cdots & 0 & 0 & 0 & 0 \\
-1 & 2 & -1 & 0 & \cdots & 0 & 0 & 0 & 0 \\
0 & -1 & 2 & -1 & \cdots & 0 & 0 & 0 & 0 \\
\vdots & & & & \ddots & & & & \vdots \\
0 & 0 & 0 & 0 & \cdots & -1 & 2 & -1 & 0 \\
0 & 0 & 0 & 0 & \cdots & 0 & -1 & 2 & -1 \\
0 & 0 & 0 & 0 & \cdots & 0 & 0 & -1 & 1
\end{array}\right)
\end{aligned}
$$

in which

$$
[\mathrm{B}]_{(\mathrm{M}-1) \times \mathrm{M}}=\left(\begin{array}{ccccccccc}
-1 & 1 & 0 & 0 & \cdots & 0 & 0 & 0 & 0 \\
0 & -1 & 1 & 0 & \cdots & 0 & 0 & 0 & 0 \\
\vdots & & & & \ddots & & & & \vdots \\
0 & 0 & 0 & 0 & \cdots & 0 & -1 & 1 & 0 \\
0 & 0 & 0 & 0 & \cdots & 0 & 0 & -1 & 1
\end{array}\right)
$$

Then, using equation $x^{2}$ [5], the minimization principle of eqn (15) is

$$
\text { minimize: } P[x]+\lambda^{(\mathrm{L})} Q[x]=|A x-b|^{2}+\lambda^{(\mathrm{L})} x \cdot H \cdot x
$$

in vector notation,

$$
\left(A^{T} A+\lambda^{(\mathrm{L})} \mathrm{H}\right) x=A^{T} b
$$

\subsubsection{The adaptive error estimation}

To obtain the optimal $\lambda$ without exact solution, the role of the adaptive tactical procedure is important to handle the inverse problem for Laplace equation with overspecified boundary condition. The method of the proposed adaptive error estimation is described as follows:

According to the ill-posed problem with the artificial contamination subjected to $\bar{u}_{1}(\underset{\sim}{x}), \underset{\sim}{x} \in B_{1}$ and $\bar{t}_{1}(\underset{\sim}{x}), \underset{\sim}{x} \in B_{1}$ as shown in Fig.2, remedied by the regularization method, we can obtain the result $\overline{\bar{u}}_{2}(\underset{\sim}{x}), \underset{\sim}{x} \in B_{2}$. Then the new 


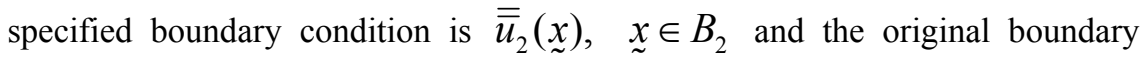
condition, $\bar{t}_{1}(\underset{\sim}{x}), \underset{\sim}{x} \in B_{1}$, in which $\overline{\bar{u}}_{2} \underset{\sim}{\underset{\sim}{x})}$ is obtained before. The new problem (well-posed) becomes the mixed-type problem as shown in Fig. 3. Furthermore, the result $\overline{\bar{u}}_{1}(\underset{\sim}{x}), \quad \underset{\sim}{x} \in B_{1}$ is calculated again by using the Trefftz method, and compare it with the original boundary condition $\bar{u}_{1}(\underset{\sim}{x}), \underset{\sim}{x} \in B_{1}$. Usually, the norm error can be defined as $\left\|\bar{u}_{1}-\overline{\bar{u}}_{1}\right\|=\left\{\int\left|\bar{u}_{1}-\overline{\bar{u}}_{1}\right|^{2} \mathrm{~d} B_{1}\right\}$, which is chosen as the index of sensitivity and $\lambda_{1}$ is chosen as the index of degree of distortion.
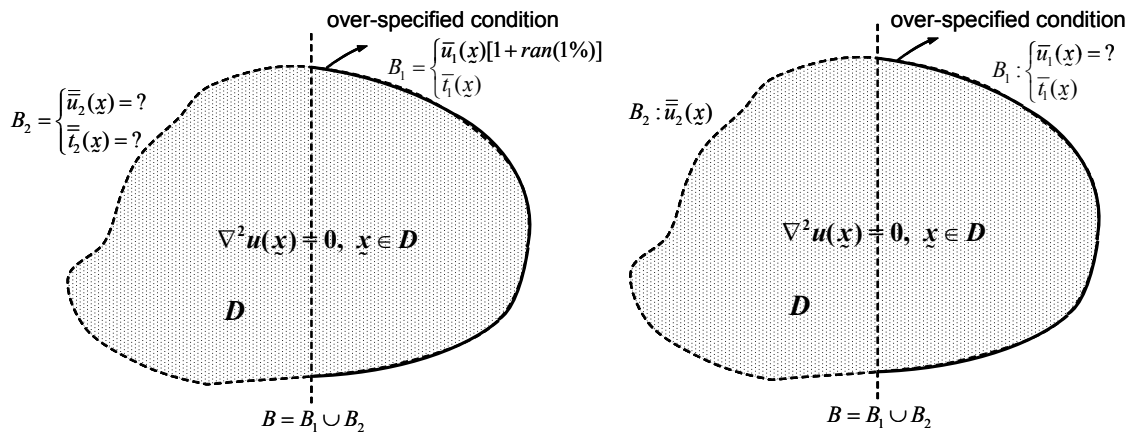

Figure 2: $\quad$ Sketch diagram of the illposed problem with the artificial contamination.

Figure 3: $\quad$ Sketch diagram of mixed-type problem with mixed-type condition.

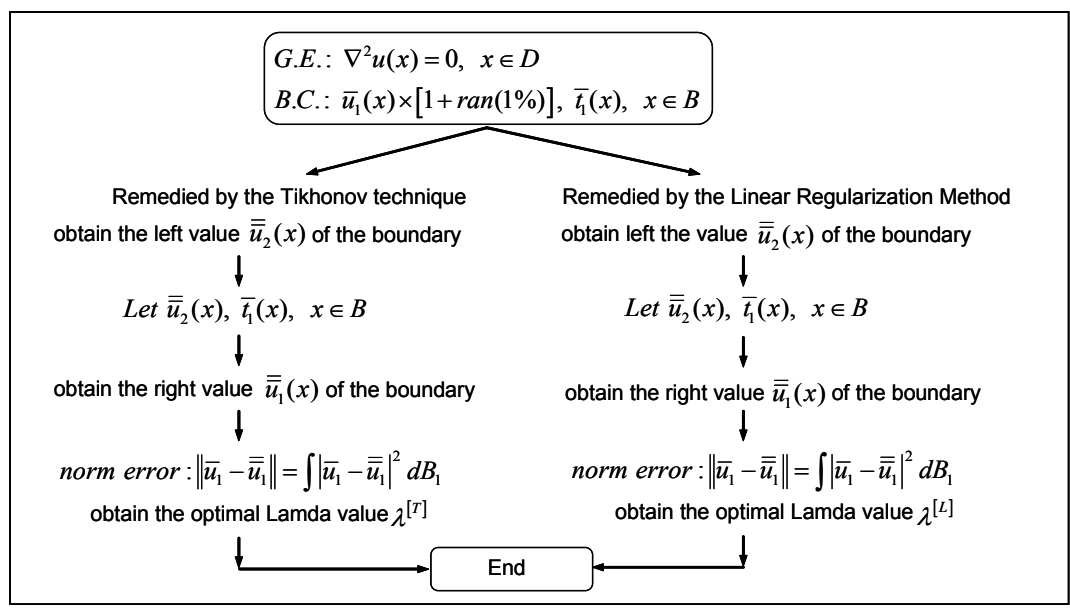

Figure 4: Flowchart of adaptive error estimation. 
In the adaptive error estimation, the L-curve shape can be observed and the optimal $\lambda$ is located on the corner. The corner is a compromise between regularization error (due to data smoothing) and perturbation errors (due to noise disturbance). To clarify the procedure, the flowchart of the adaptive error estimation can be described in Fig. 4.

\section{Numerical examples}

To illustrate applications of the Trefftz method in conjunction with the Tikhonov technique, the Linear regularization method and the adaptive error estimation for the Laplace equation with overspecified BCs, the case for a circle domain, the radius $R=1.0$ is chosen as a representation example. Two kinds of treatments in the ill-posed problem are considered: the Tikhonov technique and the Linear regularization method, all for the inverse problem contaminated by noise pollutions.

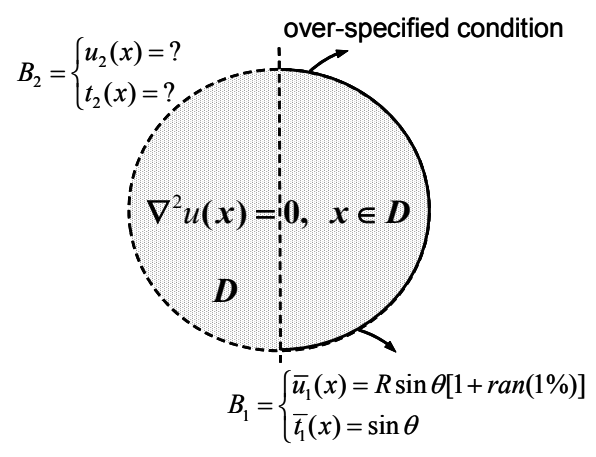

Figure 5: A sketch diagram.

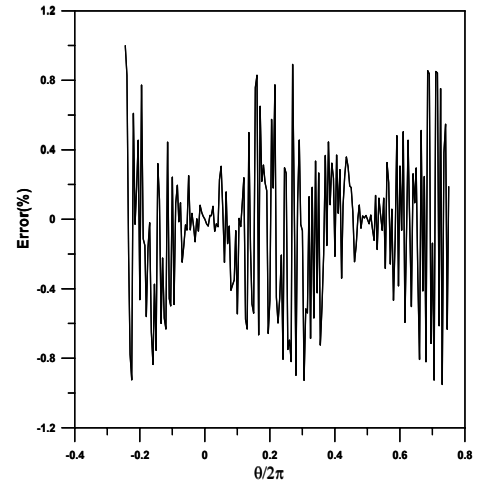

Figure 6: The random error.

\subsection{Circular case}

The present model of the inverse problem with noises can be shown as Fig. 5. By using random data simulation, we can obtain $1 \%$ random errors to contaminate the input boundary data, as described in Fig. 6. If regularization techniques are not employed, the results by using the Trefftz method are unreasonable and divergent as shown in Fig. 7(a), 7(b).

When the Tikhonov technique and the linear regularization method are applied in this case, we can obtain solutions with different values of the regularized parameters of $\lambda^{(\mathrm{T})}$ and $\lambda^{(\mathrm{L})}$ by employing the Tikhonov technique and the linear regularization method respectively, as shown in Fig. 8(a), 8(b), respectively, and the field solutions are shown in Fig. 9(a), 9(b). To obtain the optimal $\lambda$, the norm error comparing with exact solution is defined as $\left\|u-u_{e}\right\|=\left\{\int_{a}^{b}\left|u-u_{e}\right|^{2} \mathrm{~d} x\right\}$ and the norm errors versus $\lambda$ are plotted in Fig. 10, 
by using the Tikhonov technique and the linear regularization method respectively. The L-curve shape can be observed and the optimal $\lambda$ is located on the corner as shown in Fig. 11(a), 11(b).

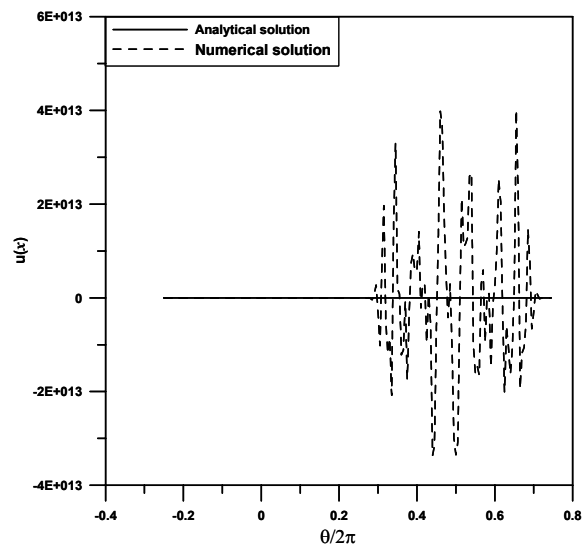

(a)

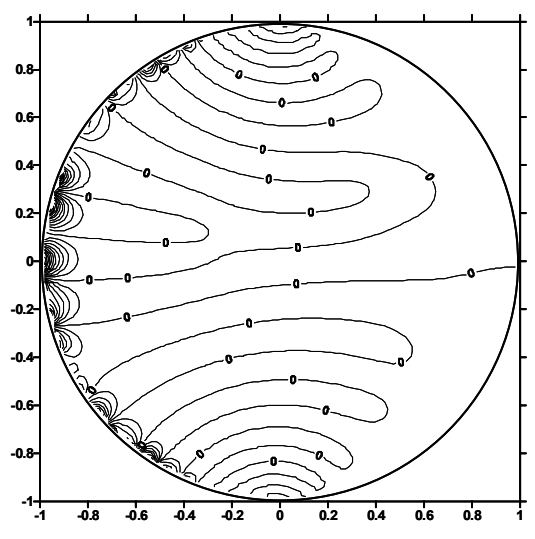

(b)

Figure 7: (a) Analytical solution and the boundary potential without regularization techniques, (b) the Field solution without regularization techniques.

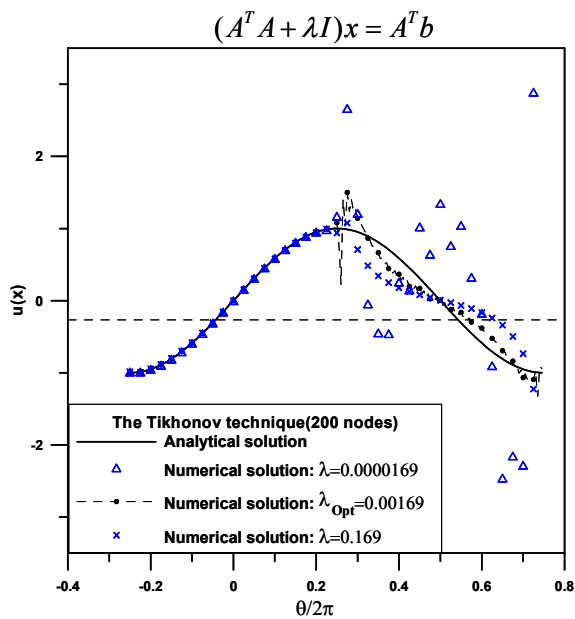

(a)

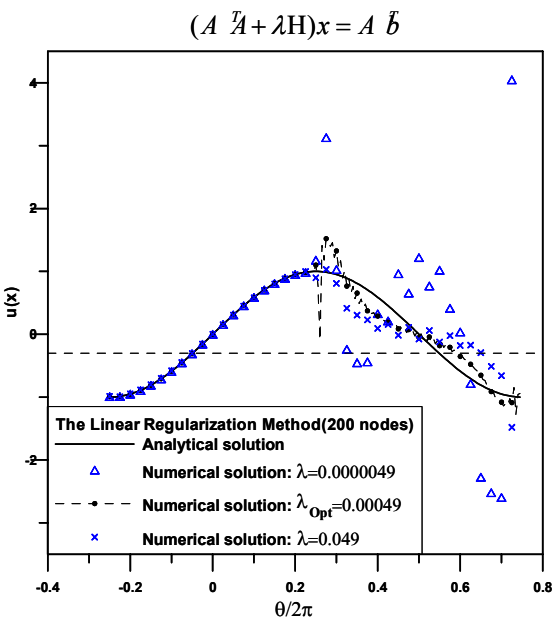

(b)

Figure 8: The numerical solution remedied by (a) the Tikhonov technique and (b) the linear regularization method with 3 different lambdas (200 nodes). 

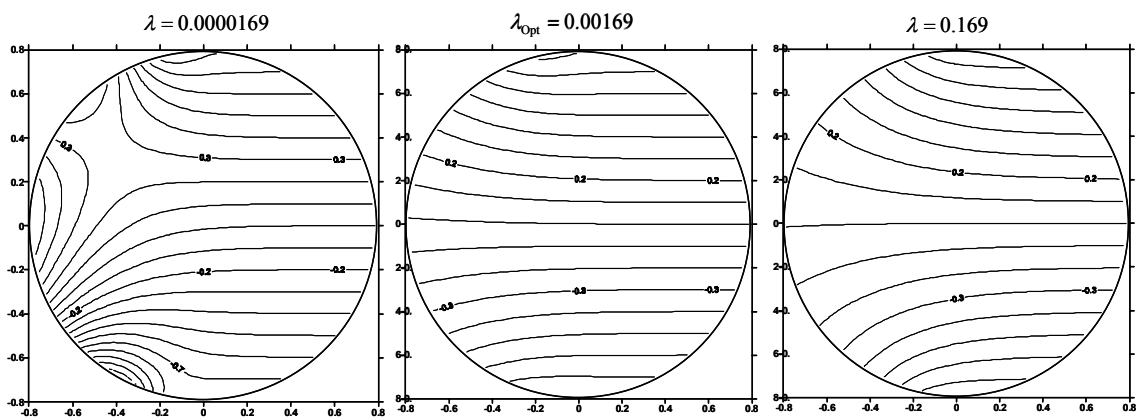

(a)
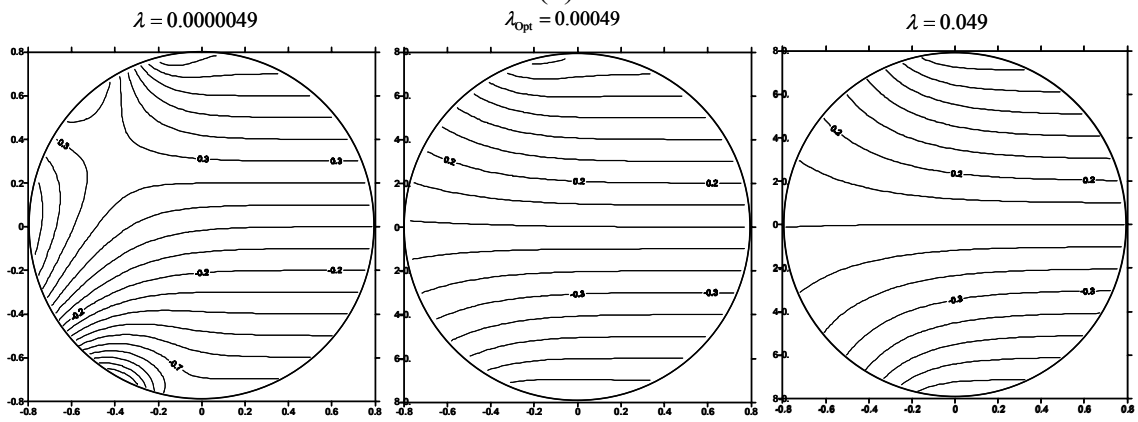

(b)

Figure 9: (a) The numerical field solution remedied by the Tikhonov technique and the linear regularization method with 3 different lambdas (200 nodes), (b) the numerical field solution remedied by the linear regularization method with 3 different lambdas (200 nodes).

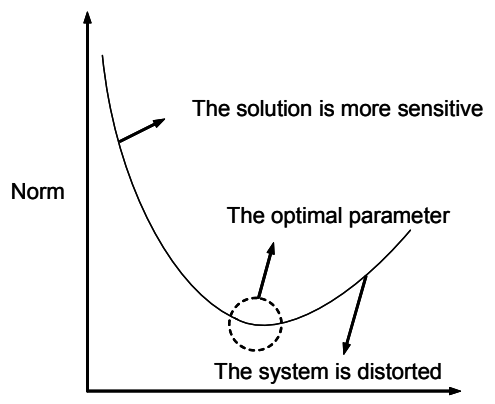

Figure 10: A sketch diagram.

As we expected from the mathematical point of view, a corner is presented in the L-curve shape. If the corner of the L-curve is chosen as an optimal point, the appropriate value is 0.00169 for the Tikhonov technique and 0.00049 for the linear regularization method respectively. 
Therefore, we can figure out the norm error of the L-curve shape by the Tikhonov technique is much lower than the linear regularization method shown as Fig. 12. Then the results have been regularized to approximate the analytical solution, as shown in Fig. 13, and the field solutions are shown in Fig. 14(a), 14(b). The regularized result by the Tikhonov technique is more approximate than that by the linear regularization method. However, the exact solution is needed from the definition of norm error.

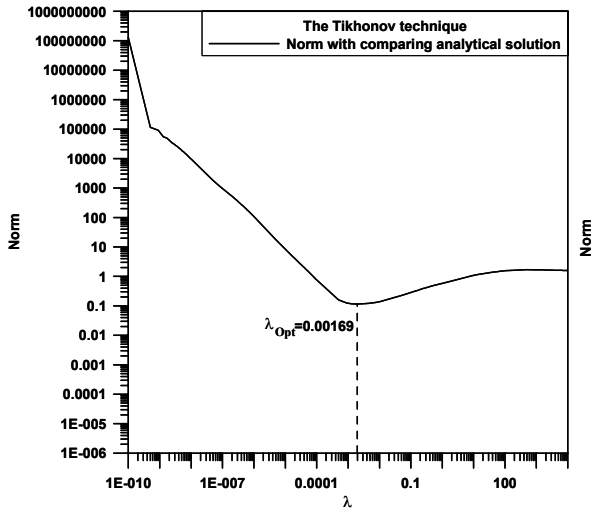

(a)

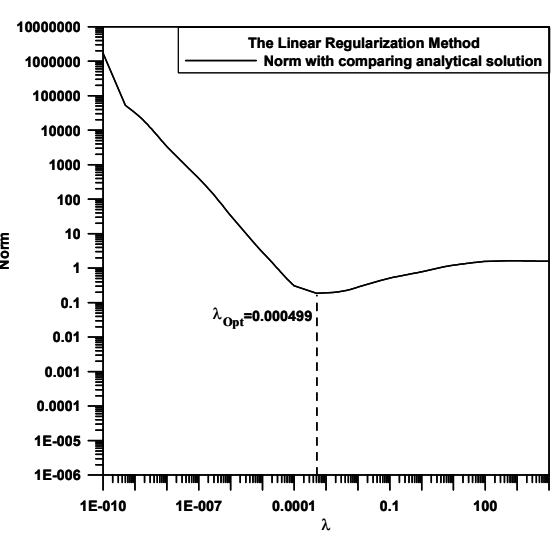

(b)

Figure 11: (a) and (b) The norm deriving from comparing numerical solution with analytical solution by the Tikhonov technique and the linear regularization method.

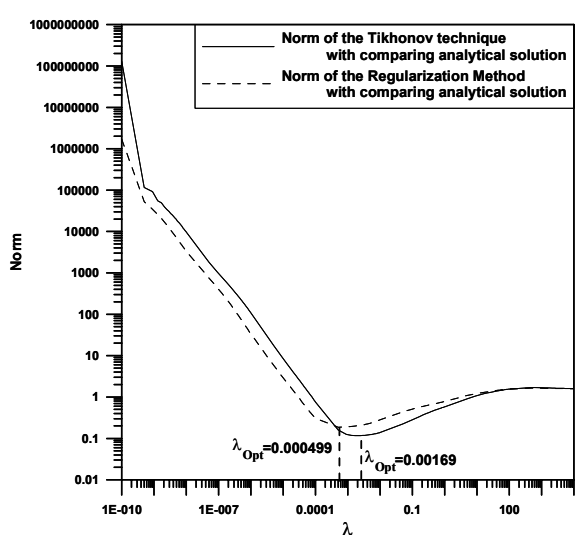

Figure 12: The norm error of the Lcurve shape by the Tikhonov technique is much lower than the linear regularization method.

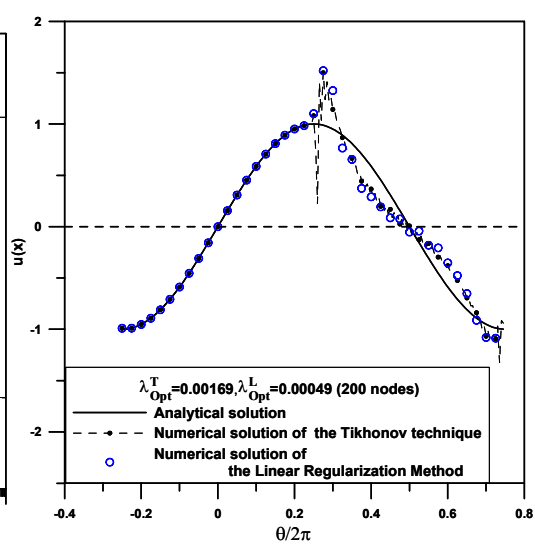

Figure 13: Numerical solution being remedied by the Tikhonov technique and the linear regularization method with the optional lambda (200 nodes). 


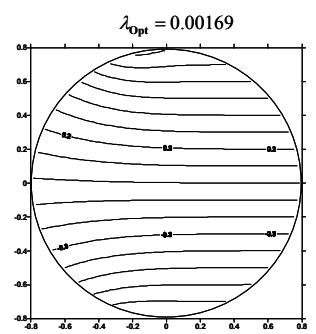

(a)

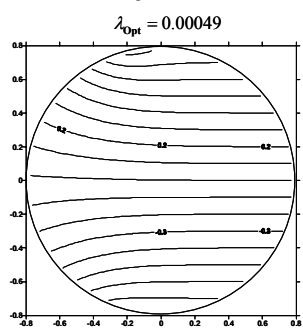

(b)

Figure 14: Numerical field solution being remedied by (a) the Tikhonov technique and (b) the linear regularization method with optional lambda (200 nodes).

We are well aware that many problems usually have no analytic solution. In order to assess the validity of the Tikhonov technique and the linear regularization method and to find out the optimal solution, choosing the adaptive error estimation without exact solution is needed. The new norm error is implemented as defined in the section 2.2.3 and obtain the optimal $\lambda$. We find the optimal $\lambda$ by implementing the adaptive error estimation is similar with the before $\lambda$, as shown in Fig. 15(a), 15(b). Therefore, we can derive the optimal result by employing the adaptive error estimation even though no exact solution can be obtained.

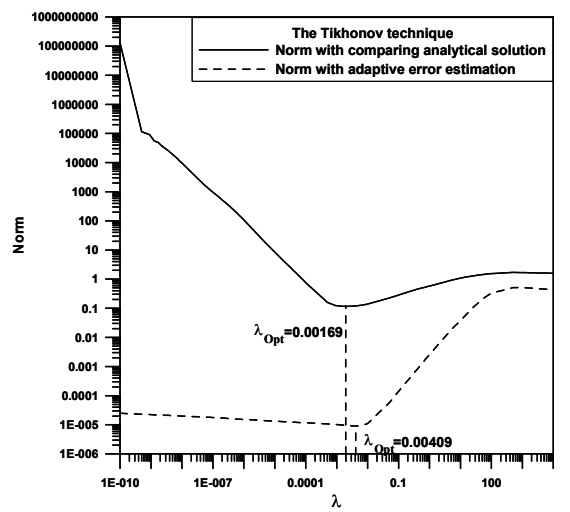

(a)

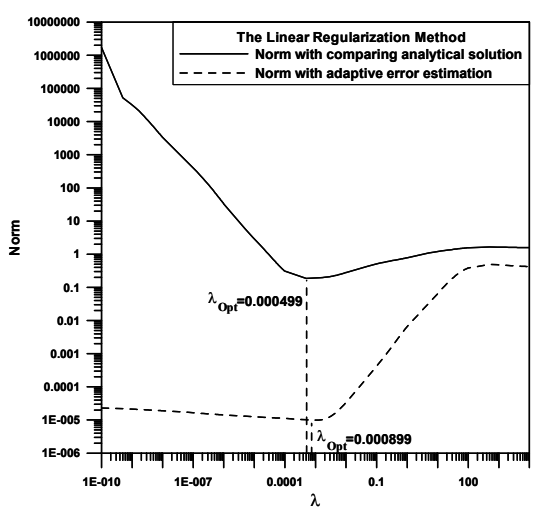

(b)

Figure 15: (a) The optimal result by employing the adaptive error estimation and the Tikhonov technique under no exact solution, (b) the optimal result by employing the adaptive error estimation and the linear regularization method under no exact solution.

\section{Conclusion}

In this paper, we used the Trefftz method to solve the Laplace equation in a twodimensional finite domain with overspecified boundary condition. 
The numerical instability existing in the solver owing to the regular formulation of this method is encountered. To overcome this difficulty, the regularization techniques using the Tikhonov technique and the linear regularization method, together with the L-curve, plays a role in determining the optimal parameter $\lambda$ which can maintain the system characteristic and can make the system insensitive to contaminating noise.

Furthermore, the numerical results obtained by using the Tikhonov technique for the case are in very close agreements with the analytical solutions, adaptive error estimation and outperform other regularization techniques.

\section{References}

[1] Tikhonov, A.N. \& Arsenin, V.Y., Solutions of Ill-posed Problems, V.H. Winston and Sons: Washington, D.C., 1977.

[2] Poluektov, A.R., Short communications: a method of choosing the regularization parameter for the numerical solution of ill-posed problems. Computational Mathematics and Mathematical Physics, 32(3), pp. 397401, 1992.

[3] Jin, B. A meshless method for the Laplace and Biharmonic equations subjected to noisy boundary data. Computer Modeling in Engineering and Sciences, 6(3), pp. 253-261, 2004.

[4] Fairweather, G. \&Andreas, K., The method of fundamental solutions for elliptic boundary value problems. Advances in Computational Mathematics, 9, pp. 69-95, 1998.

[5] William, H.P., Saul, A.T., William, T.V. \& Brian, P.F., Numerical Recipes in Fortran, Second edition, Cambridge University Press: New York; 1992.

[6] Jirousek, J. \& Wroblewski, A., T-elements: state of the art and future trends. Archives of Computational Methods in Engineering, 3-4, pp. 323434, 1996.

[7] Chang, J.R., Liu, R.F., Kuo S.R., \& Yeih, W., Application of symmetric indirect Trefftz method to free vibration problems in 2D. International Journal for Numerical Methods in Engineering, 56(8), pp. 1175-1192, 2003.

[8] Chang, J.R., Liu, R.F., Yeih, W.C. \& Kuo, S.R., Applications of the direct Trefftz boundary element method to the free-vibration problem of a membrane. Journal of the Acoustical Society of America, 112(2), pp. 518527, 2002.

[9] Chang, J.R., Yeih, W. \& Shieh, M.H., On the modified Tikhonov's regularization method for the Cauchy problem of the Laplace equation. Journal of Marine Science and Technology, 9(2), pp. 113-121, 2001.

[10] Chen, J.T., Wu, C.S., Lee, Y.T. \& Chen, K.H., On the equivalence of the Trefftz method and method of fundamental solutions for Laplace and biharmonic equations. Computers and Mathematics with Applications, (forthcoming). 
[11] Chen, J.T. \& Chen, K.H., Analytical study and numerical experiments for Laplace equation with overspecified boundary conditions. Applied Mathematical Modelling, 22, pp. 703-725, 1998.

[12] Wu, K.-L., Chen, K.-H., Chen, J.-T. \& Kao, J.-H., Regularized meshless method for solving the Cauchy problem. The $30^{\text {th }}$ National Conference on Theoretical and Applied Mechanics, Da-Yeh University: Changhwa, Taiwan, R.O.C., December 15-16, 2006

[13] Chen, L.Y., Chen, J.T., Hong, H.K. \& Chen, C.H., Application of Cesàro mean and the L-curve for the deconvolution problem. Soil Dynamics and Earthquake Engineering, 14, pp. 361-373, 1995.

[14] Tanaka, M. \& Bui, H.D., (Eds.) Inverse problems in engineering mechanics, IUTAM symposium, Springer: Berlin, 1992.

[15] Kupradze, V.D., A method for the approximate solution of limiting problems in mathematical physics. Computational Mathematics and Mathematical Physics, 4, pp. 199-205, 1964.

[16] Ivanov, V.K., The Cauchy problem for the Laplace equation in an infinite strip. Differentsial'nye Uravneniya, 1(1), pp. 131-136, 1965.

[17] Jin, W.G., Cheung, Y.K. \& Zienkiewicz, O.C., Trefftz method for Kirchoff plate bending problems. International Journal for Numerical Methods in Engineering, 36, pp. 765-781, 1993.

[18] Yeih, W., Inverse Problems in Elasticity, Ph.D. Dissertation, Northwestern University, 1991.

[19] Yeih, W., Liu, R.F., Chang, J.R. \& Kuo, S.R., Numerical instability of the direct Trefftz method for Laplace problems for a 2D finite domain. International Journal of Applied Mathematics and Mechanics, 2(1): 41 66,2006 